\title{
Supermodes of a double-ring fibre array with symmetric coupling
}

\author{
${ }^{1}$ Alexeyev C.N., ${ }^{1}$ Fadeyeva T.A., ${ }^{1,2}$ Boklag N.A. and ${ }^{1}$ Yavorsky M.A. \\ ${ }^{1}$ Taurida National V. I. Vernadsky University, 4 Vernadsky Ave., 95007 \\ Simferopol, Crimea, Ukraine, c.alexeyev@yandex.ua \\ ${ }^{2}$ National Space Facilities Control and Test Centre, 97419 Yevpatoriya, \\ Crimea, Ukraine
}

Received: 01.03 .2011

\begin{abstract}
We study the structure of supermodes of a double-ring array of identical evanescently coupled single-mode fibres with the same coupling constant, which describes interaction between the fibres. We obtain the expressions for normal modes of such an array and the spectrum of their propagation constants. We show that these supermodes are represented by symmetric and antisymmetric combinations of supermodes of a single-ring circular array.
\end{abstract}

Keywords: fibre array, circular array, double-ring array

PACS: 42.81Q; 42.81Qb

UDC: 535.32

\section{Introduction}

Since the seminal article by A. Jones [1], propagation of light in evanescently coupled fibre arrays has evoked a large amount of interest of both theorists and experimenters. At present, the physics of one- and two-dimensional infinite fibre arrays has been studied in detail in a number of works [2-4]. This is partially explained by a novel phenomenon of discrete diffraction, which takes place in waveguide arrays $[5,6]$. Some interesting results have been obtained for the curved [7] and nonlinear [8,9] waveguide arrays. Fibre arrays facilitate demonstrating a number of effects from the other fields of physics [10-12]. Moreover, they are used for creating optical beams with subwavelength resolution [13]. One can single out an important type of circular fibre array, which has been addressed in a number of studies [14-16]. A practical interest in these arrays is associated with their applications aimed at creation of cylindrical beams [17] and orbital angular momentum generation by the arrays $[18,19]$.

Nonetheless, only one type of the circular fibre arrays has been studied, a single-ring circular array. Meanwhile, it would be of great interest to go beyond the frameworks of single-ring array model. The aim of the present study is to develop a technique for obtaining the structure of supermodes in a circular array, which consists of two concentric rings each composed of identical weakly guiding single-mode fibres. We generalise a method of eigenmodes suggested earlier [20,21], on which basis we find the structure of supermodes and the spectrum of their propagation constants for the double-ring array of identi- 
cal single-mode fibres. Here we limit our consideration to the case of double-ring arrays with equal values of the constants that govern evanescent coupling between the fibres belonging to different circles and the fibres of the same circle.

\section{Supermodes of double-ring arrays}

Consider a double-ring fibre array consisting of two concentric evanescently coupled circular arrays, each of which being composed of $N$ identical single-mode fibres. The centres of the fibres are positioned at the vertices of homothetical polygons ( $N$-gons) as shown in Fig. 1. The coupling between the nearest neighbours is supposed to be symmetric and is described by the constant $J$. For step-index fibres, the coupling is given by a standard exchange integral, which may be brought to the form

$$
C=4 k^{2} \Delta n_{c o}^{2} \exp \left(-L^{2} / 2 r_{0}^{2}\right) \int_{0}^{1} r \exp \left(-r^{2}\right) J_{0}\left(i L r / r_{0}\right) d x
$$

Here $L$ denotes the distance between the centres of the fibres, the radial coordinate $r$ is associated with the centre of one of the two adjacent coupled fibres [15], $\Delta=\left(n_{c o}^{2}-n_{c l}^{2}\right) / 2 n_{c o}^{2}$ (with $n_{c o}$ and $n_{c l}$ being the refractive indices of the core and cladding, respectively), $r_{0}$ is the core radius, $k$ the wave number in vacuum, and $J_{0}$ the zero-order Bessel function. To obtain Eq. (1), we have assumed that the field $\Psi$ of the fundamental mode is represented in the Gaussian approximation as [15]

$$
\Psi=E \exp \left(-\frac{r^{2}}{2 r_{0}^{2}}\right),
$$

where $E$ is some constant. Strictly speaking, such a symmetric exchange cannot be realised in the system; however, it seems to be a good approximation if the distance between the circles is much less than the radius of the inner ring. When studying the modes of the array, we are to construct the perturbation matrix of the total Hamiltonian-like operator that describes propagation of light in it.

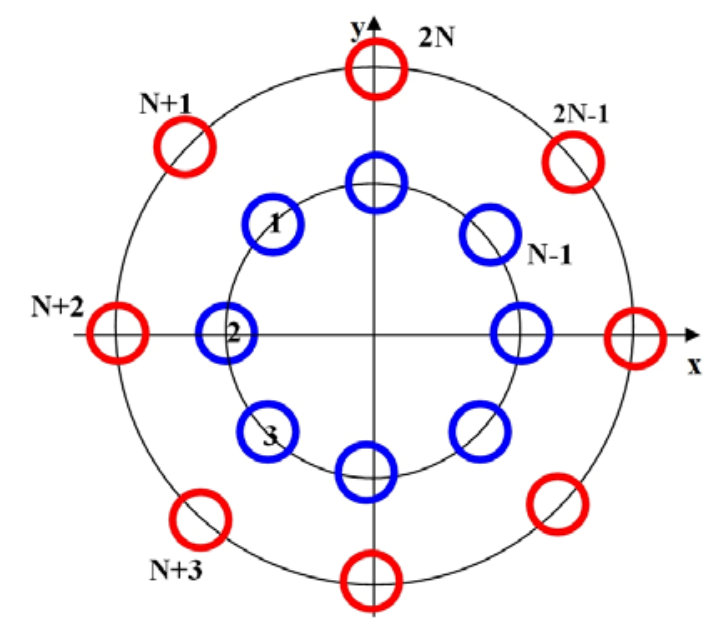

Fig.1.(colour online). Schematic view (transverse cross-section) of a double-ring array and scheme of numeration of fibres in the array. 
In the scalar approximation, propagation of light in the array of step-index fibres is governed by the waveguide equation

$$
\left(\nabla_{t}^{2}+k^{2} n_{c l}^{2}+V\right) \vec{e}_{t}=\tilde{\beta}^{2} \vec{e}_{t}
$$

where $\vec{\nabla}_{t}=(\partial / \partial x, \partial / \partial y), \tilde{\beta}$ is the propagation constant, and $\vec{e}_{t}$ the transverse part of the electric field introduced as $\vec{E}(x, y, z)=\vec{e}(x, y) e^{i \tilde{\beta} z}$. The perturbation $V$ may be written as $V=2 k^{2} n_{c o}^{2} \Delta \sum_{i} \delta\left(r_{0}-r_{i}\right)$. It is assumed here that the $i$-th fibre is associated with a local polar coordinate system, in which the $r_{i}$ coordinate is defined. For infinitely spaced fibres, the solution of Eq. (3) may be represented as a linear superposition of scalar solutions $\psi(r)$ localised at the $i$-th fibre: $\left|e_{t}\right\rangle=\sum_{i} c_{i}|i\rangle$, with $|i\rangle=\operatorname{col}(0,0 \ldots \psi(r) \ldots 0,0)$ and $\psi$ standing for the $i$-th place. As the separation of fibres becomes less, the modes of the individual fibres get combined and form some unknown linear combinations, which are conventionally called as supermodes. For obtaining the structure of such supermodes one can apply a standard procedure of perturbation theory in the presence of degeneracy, which is widely accepted in quantum mechanics [18] and has been developed for the coupled fibres in the works [20,21,23]. According to it, one should average Eq. (3) over the basis $\{|i\rangle\}$ and determine the structure of supermodes on the basis of eigenvectors of the matrix obtained. As can be shown (see [20]), the matrix obtained in the nearestneighbour approximation for the one-dimensional infinite array of identical fibres has the following elements:

$$
H_{i k}=\tilde{\beta}^{2} \delta_{i k}+C\left(\delta_{i, k+1}+\delta_{i+1, k}\right),
$$

where $\delta_{i k}$ is the Kronecker delta. Imposing periodic boundary conditions $|i\rangle=|i+N\rangle$, one can describe a circular array of $N$ fibres with the matrix elements given by Eq. (4). The first term on the r. h. s. of Eq. (4) is usually omitted since it does not affect the form of the eigenvectors. Here we will also omit dependence of the $\tilde{\beta}$ constant on the radial number $m$.

The dimension of the $H$-matrix for the double-ring array of $2 N$ fibres equals to $2 N$. It is easily verified that in order to numerate the fibres as in Fig. 1, one should choose the following expression for the corresponding perturbation matrix $H_{2 N}$ that determines the structure of the modes:

$$
H_{2 N}=J\left(\begin{array}{cc}
M_{N} & 1_{N} \\
1_{N} & M_{N}
\end{array}\right),
$$

where $1_{N}$ represents the unity matrix of the rank $N$ and $\left(M_{N}\right)_{i k}=\delta_{i, k+1}+\delta_{i+1, k}$. It is evident that the matrix $M_{N}$ just describes the circular fibre array. The problem of finding the supermodes of the double-ring array is equivalent to the problem of diagonalisation of 
the matrix $H_{2 N}$. To this end one should recall that the matrix $M_{N}$ is diagonalised by the lattice Fourier transform expressed in terms of diagonalising unitary transformation:

$$
\tilde{M}_{N}=U_{N} M_{N} U_{N}^{+},
$$

where the elements of rank-N matrix $U_{N}$ are given by $\left(U_{N}\right)_{k l}=\frac{1}{\sqrt{N}} \exp \left(i \varphi_{k} l\right)$ $\left(\varphi_{k}=\frac{2 \pi k}{N}\right)$. Defining a rank-2N matrix $C_{2 N}=\left(\begin{array}{cc}U_{N} & 0_{N} \\ 0_{N} & U_{N}\end{array}\right)$, where a rank- $N$ matrix $0_{N}$ has zero elements, one obtains

$$
C_{2 N} H_{2 N} C_{2 N}^{+}=J\left(\begin{array}{cc}
D_{N} & 1_{N} \\
1_{N} & D_{N}
\end{array}\right) .
$$

Here a diagonal matrix $D_{N}$ is composed of the spectrum of the circular array of $N$ fibres, so that we have

$$
\left(D_{N}\right)_{n k}=2 \delta_{n k} \cos \varphi_{n}
$$

Note that no summation over $n$ is implied here.

In order to diagonalise the matrix given by Eq. (7), let us introduce the matrix $L_{2 N}=L_{2 N}{ }^{+}=\frac{1}{\sqrt{2}}\left(\begin{array}{cc}1_{N} & 1_{N} \\ 1_{N} & -1_{N}\end{array}\right)$. Using direct calculations, one can easily testify that

$$
L_{2 N} C_{2 N} H_{2 N} C_{2 N}{ }^{+} L_{2 N}{ }^{+}=J\left(\begin{array}{cc}
D_{N}+1_{N} & 0_{N} \\
0_{N} & D_{N}-1_{N}
\end{array}\right) .
$$

Since the matrices $D_{N} \pm 1_{N}$ are diagonal, the spectrum of the supermodes looks like

$$
\beta_{m}^{2}=\tilde{\beta}^{2}+J\left(2 \cos \varphi_{m} \pm 1\right)
$$

Notice also that the relation $\beta_{m}=\tilde{\beta}+\frac{J}{2 \tilde{\beta}}\left(2 \cos \varphi_{m} \pm 1\right)$ follows from Eq. (10) [15].

The structure of the eigenmodes can be obtained through the following standard procedure. The state of the double-ring array is described by a $2 N$-dimensional vector $\vec{X}_{2 N}=\operatorname{col}\left(q_{1} \ldots q_{N}, p_{1} \ldots p_{N}\right) \equiv \operatorname{col}\left(\vec{Q}_{N}, \vec{P}_{N}\right)$, where $q_{i}$ describes the amplitude for the $i$-th fibre in the inner ring, and $p_{i}$ stands for the amplitude for the $i$-th outer-ring fibre. It is obvious that the eigenmodes of the system are given by the elements of the vector $L_{2 N} C_{2 N} \vec{X}_{2 N}$. It is convenient to express these eigenmodes in terms of the eigenmodes of the inner and outer rings ( $X_{m}$ and $Y_{m}$, respectively), if they are taken separately [19]:

$$
\begin{aligned}
& X_{m}=\frac{1}{\sqrt{N}} \sum_{k=0}^{N-1} \exp \left(i \varphi_{m} k\right) q_{k}, \\
& Y_{m}=\frac{1}{\sqrt{N}} \sum_{k=0}^{N-1} \exp \left(i \varphi_{m} k\right) p_{k}
\end{aligned}
$$


with $|m|=0,1 \ldots N-1$. After a little algebra, the expressions for the modes can be obtained in the following form:

$$
Z_{m}^{( \pm)}=\frac{1}{\sqrt{2}}\left(X_{m} \pm Y_{m}\right)
$$

This form conveys the idea of in-phase and out-of-phase propagations of the supermodes related to the inner and outer rings.

Eqs. (10)-(12) solve the problem of the structure and spectrum of the supermodes for the double-ring array. The results obtained may be used while investigating the problems concerned with light propagation in the appropriate optical systems, in particular the problems of light channelling and discrete diffraction in circular arrays, and a Talbot effect occurring in the double-ring arrays. The mathematical method developed by us can also be applied to study more realistic models of the array assuming an asymmetric exchange.

\section{Conclusion}

In this work we have developed a technique of normal modes in order to study the structure of supermodes in a double-ring array of identical single-mode fibres. By using the perturbation theory with degeneracy, we have obtained the expressions for the supermodes of such an array and the spectrum of their propagation constants. We have shown that these supermodes are given by symmetric and antisymmetric combinations of the supermodes characteristic of a single-ring circular array.

\section{References}

1. Jones A L, 1965. Coupling of optical fibers and scattering in fibers. J. Opt. Soc. Amer. 55: 261-269.

2. Szameit A, Pertsch T, Dreisow F, Nolte S and Tünnermann A, 2007. Light evolution in arbitrary two-dimensional waveguide arrays. Phys. Rev. A. 75: 053814.

3. Efremidis N K, Zhang P, Chen Z, Christodoulides D N, Rüter C E and Kip D, 2010. Wave propagation in waveguide arrays with alternating positive and negative couplings. Phys. Rev. A. 81: 053817.

4. Patra K C, Srivastava S and Sharma E K, 2010. Power exchange in strongly coupled diffused channel waveguide arrays: an analytical approach. J. Opt. 12: 08550.

5. Christodulides DN, Lederer F, Silberberg Y, 2003. Discretizing light behaviour in linear and nonlinear waveguide lattices. Nature. 424: 817-823.

6. Longhi S, 2009. Discrete diffraction and shape-invariant beams in optical waveguide arrays. Phys. Rev. A. 79: 033847.

7. Longhi S, 2009. Rectification of light refraction in curved waveguide arrays. Opt. Lett. 34: 458-460.

8. Kartashov Y V, Malomed B A, Vysloukh V A and Torner L, 2009. Stabilization of multibeam necklace solitons in circular arrays with spatially modulated nonlinearity. Phys. Rev. A. 80: 053816.

9. Setzpfandt F, Sukhorukov A A, Neshev D N, Schiek R, Kivshar Y S and Pertsch T, 
2010. Phase transitions of nonlinear waves in quadratic waveguide arrays. Phys. Rev. Lett. 105: 233905.

10. Longhi S, 2009. Polychromatic optical Bloch oscillations. Opt. Lett. 34: 2174-2176.

11. Thompson C, Vemuri G and Agarwal G S, 2010. Anderson localization with second quantized fields in a coupled array of waveguides. Phys. Rev. A. 82: 053805.

12. Khomeriki R, 2010. Nonlinear Landau-Zener tunneling in coupled waveguide arrays. Phys. Rev. A. 82: 013839.

13. Wang $\mathrm{X}, \mathrm{Fu}$ J, Liu $X$ and Tong L-M, 2009. Subwavelength focusing by a micro/nanofiber array. J. Opt. Soc. Amer. A. 26: 1827-1833.

14. Snyder A W, 1972. Coupled-mode theory for optical fibers. J. Opt. Soc. Amer. 62: $1267-1277$.

15. Snyder A W and Love J D, Optical waveguide theory. London, New York: Chapman and Hall (1985).

16. Longhi S, 2007. Light transfer control and diffraction management in circular fibre waveguide arrays. J. Phys. B. 40: 4477.

17. Kurti R S, Halterman K, Shori R K and Wardlaw M J, 2009. Discrete cylindrical vector beam generation from an array of optical fibers. Opt. Express. 17: 13982-13988.

18. Yu Y F, Fu Y H, Zhang X M, Liu A Q, Bourouina T, Mei T, Shen Z X and Tsai D P, 2010. Pure angular momentum generator using a ring resonator. Opt. Express. 18: 21651-21662.

19. Alexeyev C N, Volyar A V and Yavorsky M A, 2009. Linear azimuthons in circular fiber arrays and optical angular momentum of discrete optical vortices. Phys. Rev. A. 80: 063821.

20. Alexeyev C N, Alexeyev A N, Boklag N A and Yavorsky M A, 2009. Effect of the spin-orbit interaction on polarization conversion in coupled waveguides. J. Opt. A: Pure Appl. Opt. 11: 125404.

21. Alexeyev C N, Boklag N A and Yavorsky M A, 2010. Higher order modes of coupled optical fibres. J. Opt. 12: 115704.

22. Davydov A S, Quantum mechanics. Oxford: Pergamon (1976).

23. Alexeyev C N, Boklag N A, Fadeyeva T A and Yavorsky M A, 2010. Tunnelling of orbital angular momentum in parallel optical waveguides. J. Opt. 13 (in press).

Alexeyev C. N., Fadeyeva T. A., Boklag N. A. and Yavorsky M. A., 2011. Supermodes of a double-ring fibre array with symmetric coupling Ukr.J.Phys.Opt. 12: 83-88.

Анотація. Досліджено структуру супермод двокільчевого джгута ідентичних, радіачійно зв'язаних одномодових волокон з однаковим значенням константи зв'язку, що описує взаємодію між волокнами. Отримано вираз для нормальних мод иього джгута $і$ спектр констант поширення. Показано, що його супермоди можна представити симетричною та антисиметричною комбіначією супермод однокільцевого круглого джгута. 